\title{
Do Cardiac Rehabilitation Affect Clinical Prognoses Such as Recurrence, Readmission, Revascularization, and Mortality After AMI?: Systematic Review and Meta-Analysis
} Chul Kim, MD, $\mathrm{PhD}^{1}$, Insun Choi, $\mathrm{PhD}^{2}$, Songhee Cho, $\mathrm{PhD}^{2}$, Ae Ryoung Kim, $\mathrm{MD}^{3}$, Wonseok Kim, $\mathrm{MD}, \mathrm{PhD}^{4}$, Sungju Jee, $\mathrm{MD}, \mathrm{PhD}^{5,6,7}$

${ }^{1}$ Department of Rehabilitation Medicine, Inje University Sanggye Paik Hospital, Seoul;

${ }^{2}$ National Evidence-based Healthcare Collaborating Agency, Seoul;

${ }^{3}$ Department of Rehabilitation Medicine, Kyungpook National University Hospital, Daegu;

${ }^{4}$ Department of Rehabilitation Medicine, Seoul National University Bundang Hospital, Seongnam;

${ }^{5}$ Department of Rehabilitation Medicine, Chungnam National University College of Medicine, Daejeon;

${ }^{6}$ Daejeonchungcheong Regional Medical Rehabilitation Center, Chungnam National University Hospital, Daejeon;

${ }^{7}$ Dajeonchungcheong Regional Cardiocerebrovascular Center, Chungnam National University Hospital, Daejeon, Korea

Objective We conducted a systematic review and meta-analysis to analyze the effects of cardiac rehabilitation (CR) on post-discharge prognoses of patients with acute myocardial infarction (AMI).

Methods A literature search was conducted through four international medical and two Korean databases. Primary outcomes for the effectiveness of CR included all-cause mortality, cardiovascular mortality, recurrence, revascularization, major adverse cardiovascular event, major adverse cardiocerebrovascular event, and readmission. We summarized and analyzed results of studies about CR for AMI, including not only randomized controlled trials (RCTs) but also non-RCTs. We calculated the effect size separately by the study type.

Results Fourteen articles were finally selected. Of these, two articles were RCTs, while 12 were non-RCTs. In RCTs, the overall mortality rate was lower in the group that participated in CR than that in the conventional care group by $28 \%$ (relative risk $=0.72 ; 95 \%$ confidence interval, $0.34-1.57$ ). Among non-RCTs, CR participation significantly decreased the overall risk of mortality. Moreover, the rates of recurrence and major adverse cardiovascular events were lower in the group that participated in CR compared to those in the non-CR group.

Conclusion The meta-analysis shows that CR reduces the risk of re-hospitalization and all-cause mortality after AMI, compared to no participation in CR. This outcome was seen in RCTs as well as in non-RCTs. More studies are necessary for concrete conclusions about the beneficial effects of CR after AMI in various settings.

Keywords Myocardial infarction, Cardiac rehabilitation, Recurrence, Readmission, Mortality

Received April 23, 2020; Revised June 30, 2020; Accepted August 7, 2020; Published online February 9, 2021

Corresponding author: Sungju Jee

Department of Rehabilitation Medicine, Chungnam National University College of Medicine, 282 Munhwa-ro, Jung-gu, Daejeon 35015, Korea. Tel: +82-42-338-2423, Fax: +82-42-338-2461, E-mail: drjeesungju@cnuh.co.kr

ORCID: Chul Kim (https://orcid.org/0000-0001-8223-2945); Insun Choi (https://orcid.org/0000-0002-5115-5171); Songhee Cho (https://orcid. org/0000-0003-2192-7182); Ae Ryoung Kim (https://orcid.org/0000-0003-3765-3024); Wonseok Kim (https://orcid.org/0000-0002-1199-5707); Sungju Jee (https://orcid.org/0000-0002-9400-9609).

(c) This is an open-access article distributed under the terms of the Creative Commons Attribution Non-Commercial License (http://creativecommons.org/ licenses/by-nc/4.0) which permits unrestricted noncommercial use, distribution, and reproduction in any medium, provided the original work is properly cited. Copyright ( 2021 by Korean Academy of Rehabilitation Medicine 


\section{INTRODUCTION}

The rate of mortality from cardiovascular causes in South Korea has rapidly increased by $41.6 \%$, over a 10 year period between 2005 and 2015, and has become the second most common cause of death since 2014. Among the causes of cardiac death, ischemic heart disease accounted for approximately 52\%; 28.9 per 100,000 individuals died of this disease in 2015, and in most cases, the cause of death was acute myocardial infarction (AMI) [1].

Following the treatment of AMI, the patients' physical quality of life deteriorated; however, the awareness about cardiac rehabilitation (CR) remained low [2]. There has been a lot of evidence supporting the importance of $\mathrm{CR}$, and it has been suggested that the secondary prevention should begin soon after the acute stage of the illness, to minimize the prolonged inactivity and onset of AMI-related complications [3]. Secondary prevention should include optimal medical therapy for coronary artery disease and combination therapy with appropriate medications if there are comorbidities, such as diabetes, hypertension, and hyperlipidemia. The patients should also adopt strict self-care practices that include cessation of smoking, dietary modifications, regular exercise, and stress management. However, despite receiving professional acute care during AMI, patients might face difficulties in performing regular exercises due to tachycardia, frequent arrhythmia, decreased cardiac output, frequent orthostatic hypotension, and exercise-induced hypertension for a certain period after discharge from hospital. In particular, in patients with a history of chronic ischemic heart disease who receive inpatient treatment for AMI, the overall ability to perform exercise further reduces, due to skeletal muscle degeneration and reduced muscle oxidative capacity. Although long-term CR is necessary for patients with AMI, the participation rate in CR still low, even in the Western countries, such as United States and Canada.

Anderson et al. [4] reported an updated systematic review from their previous Cochrane report. They explored the effects of exercise-based CR in patients with coronary heart disease (CHD) in terms of mortality, morbidity, health-related quality of life, and cost-effectiveness. They searched randomized controlled clinical trials published between December 2009 to July 2014. Target populations were patients' post-myocardial infarction or revascu- larization, or those with a diagnosis of angina pectoris or CHD, as defined by angiography. A total of 63 studies with 14,486 participants were included. The metaanalysis showed a reduction in cardiovascular mortality (relative risk $[\mathrm{RR}]=0.74 ; 95 \%$ confidence interval $[\mathrm{CI}]$, 0.64-0.86) and risk of hospital admission ( $\mathrm{RR}=0.82 ; 95 \%$ $\mathrm{CI}, 0.70-0.96)$. There was no significant effect on all-cause mortality, myocardial infarction (MI), or revascularization. The study confirmed consistent benefits across patients and intervention types. Moreover, these benefits were independent of the study quality, setting, and publication date.

Rauch et al. [5] explored the effect of multi-component CR on various prognoses including all-cause mortality and other clinical endpoints, after an acute coronary event. In this systematic review, they searched several randomized controlled trials (RCT), and cohort studies published in 1995 or later. They performed a meta-analysis of 1 RCT and 24 cohort studies according to the type of study, and concluded that CR participation after acute coronary syndrome (ACS) and coronary artery bypass graft (CABG) is associated with reduced mortality, even in the modern era of coronary artery disease (CAD) treatment.

In the previous systematic reviews and meta-analyses, the population comprised those with ACS including nonST elevation MI, ST-elevation MI, and unstable angina $[4,5]$. However, the most important candidates for CR could be MI patients regardless of the ST status. Due to direct injury to the myocardial muscle, MI could be a more complicated disease in the ACS spectrum compared to stable or unstable angina. However, there has been no systematic review about the effects of CR, including only patients of MI.

\section{MATERIALS AND METHODS}

The criteria for identifying and selecting study reports, outcomes of interest, methods of data extraction, methods for assessing the risk of bias and methods for statistical analysis were prespecified. We conducted and reported this systematic review in accordance with the Preferred Reporting Items for Systematic Reviews and Meta-analyses statement (PRISMA). 


\section{Data searches and sources}

The Medline, Embase, CENTRAL (Cochrane Central Register of Controlled Trials), Cumulative Index to Nursing and Allied Health Literature (CINAHL), KoreaMed, and Kmbase databases were searched. To improve the sensitivity of the search, Medical Subject Headings (MeSH) were used. Detailed searching strategies are described in Supplement A.

\section{Study selection}

The key question that was decided based on a discussion about previous studies and expert opinion was, "Can CR have an impact on recurrence, readmission, reintervention, and mortality after acute myocardial infarction?" Table 1 describes the detailed strategies for study inclusion such as population, intervention/comparator, outcomes, time, setting, and design. The outcome parameters for effectiveness included the recurrence rate, re-intervention rate, readmission rate, ischemic event, major adverse cardiac event (MACE), major adverse cardiac and cerebrovascular event (MACCE), emergency room visit, and mortality. We selected studies published after early 2000s since there have been significant changes in acute medical treatment such as percutaneous coronary intervention (PCI) including stents for AMI as previous studies did [5]. Since we narrowed down the study population to include only patients with AMI, we included not only RCTs but also non-RCTs. CR was defined as a supervised or unsupervised inpatient, outpatient, or center-based intervention that included some form of

Table 1. Strategy for study inclusion

\begin{tabular}{ll}
\hline \multicolumn{1}{c}{ Contents } \\
\hline $\begin{array}{l}\text { Population } \\
\text { comparator }\end{array}$ & Acute myocardial infarction \\
Outcomes & $\begin{array}{c}\text { Recurrence, readmission, } \\
\text { re-intervention, death }\end{array}$ \\
Time & $\begin{array}{l}\text { Publication: after 2000 } \\
\text { Duration of study and follow-up: } \\
\text { no limitation }\end{array}$ \\
Setting & RCT \\
Study design & $\begin{array}{l}\text { Non-RCT (quasi-RCT, case-control } \\
\text { study, cohort study) }\end{array}$ \\
\hline
\end{tabular}

RCT, randomized controlled trial. exercise training, either alone or in addition to psychosocial and/or educational interventions. The comparator group included those who received standard medical care, but no structured CR treatment. We included patients who had suffered a MI, irrespective of sex or age, and had undergone revascularization (CABG or PCI). We excluded those with angina pectoris or unstable anginaCHD defined by angiography. Finally, we selected studies that reported one or more of the following outcomes: mortality, recurrence, revascularization (CABG or PCI), and hospitalization. Two reviewers (S. Jee and C. Kim) independently assessed all identified titles/abstracts for possible inclusion, with any disagreements resolved by discussion or consultation with the third reviewer.

\section{Data extraction and management}

One reviewer (I. Choi) extracted the study and patient characteristics, intervention and comparator details, and outcome data from included studies using a standardized data collection form. The second and third authors (I. Choi and S. Cho) checked the data for accuracy, and disagreements were resolved by consensus. Duplicate publications of the same study were assessed for additional data and authors were contacted where necessary, to obtain additional information.

\section{Assessment of risk of bias and overall quality of evidence}

The risk of bias of included RCT and non-RCT studies was assessed using the Cochrane Collaboration's core risk of bias items and risk of bias for non-randomized studies (RoBANS), respectively [6]. GRADE guidelines were used to assess the overall quality of evidence for each outcome [7].

\section{Data synthesis and analysis}

Data were extracted for several categories, including study characteristics (design, country, duration, inclusion criteria), patient characteristics (numbers, disease categories, type of intervention, age, the composition of study and control group, place, duration of intervention, frequency), and clinical outcomes. Data from RCTs and non-RCTs were analyzed separately. If a quantitative measurement was possible, we conducted a metaanalysis and confirmed the heterogeneity, else it was described qualitatively. We used the outcome values at 
the longest follow-up time point of each study. Dichotomous outcomes were expressed as RRs with 95\% CIs. Variance and heterogeneity among the included studies were explored by using the forest plots and $\mathrm{I}^{2}$ statistics, respectively. Data from each study were pooled using a fixed-effects meta-analysis model and for the analysis

Table 2. Category and parameters for subgroup analysis

\begin{tabular}{|c|c|}
\hline Category & Parameters \\
\hline $\begin{array}{l}\text { Patient } \\
\text { characteristics }\end{array}$ & Age, sex, country \\
\hline Study design & $\begin{array}{l}\text { Number of subjects } \\
\text { Prospective or retrospective } \\
\text { Publication year }\end{array}$ \\
\hline Follow-up length & Above/below 1 year \\
\hline Risk of bias & $\begin{array}{l}\text { RCT } \\
\text { - Blindness for intervention: low/ } \\
\text { high/not known } \\
\text { - Blindness for evaluation: low/high/ } \\
\text { not known } \\
\text { Non-RCT } \\
\text { - Study population selection: low/ } \\
\text { high/not known } \\
\text { - Blindness for evaluation: low/high/ } \\
\text { not known }\end{array}$ \\
\hline
\end{tabular}

RCT, randomized controlled trial. with $\mathrm{I}^{2}$ above $75 \%$ by using the random-effects model. If there was statistical heterogeneity, meta-regression was conducted to explore the covariance that affected the random effects, and to confirm the reason for heterogeneity. Subgroup analysis was conducted according to age group and follow-up length (Table 2). All statistical analyses were performed using Review Manager (RevMan) 5.3 software (https://training.cochrane.org/online-learning/ core-software-cochrane-reviews/revman).

\section{RESULTS}

\section{Selection and inclusion studies}

Our literature search yielded 11,020 titles, of which 74 full papers were considered for inclusion. Fourteen publications were included (see Fig. 1 for a summary of the study selection process). Among 14 studies, 2 were RCTs $[8,9]$ and the others were non-RCTs (8 prospective cohort studies [10-17] and 4 retrospective cohort studies [18$21])$. Majority of the studies were conducted in Europe (64\%) or North America (22\%). The most frequent followup period was 1 year (57\%) and the longest follow-up was above 10 years (14\%). Table 3 describes both lists and characteristics of the finally selected studies. Table 4 describes variables according to the study design.

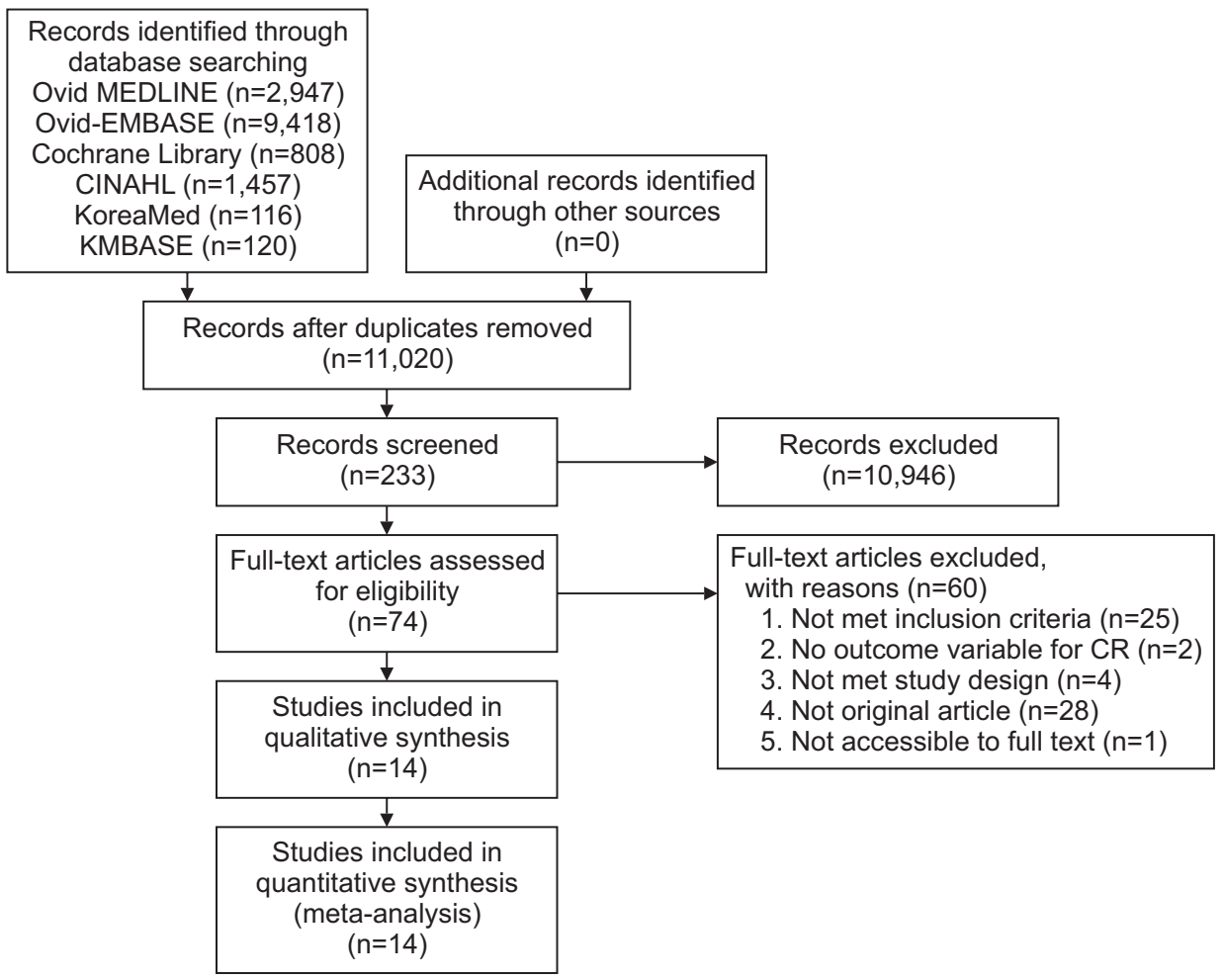

Fig. 1. Summary of study selection process. 


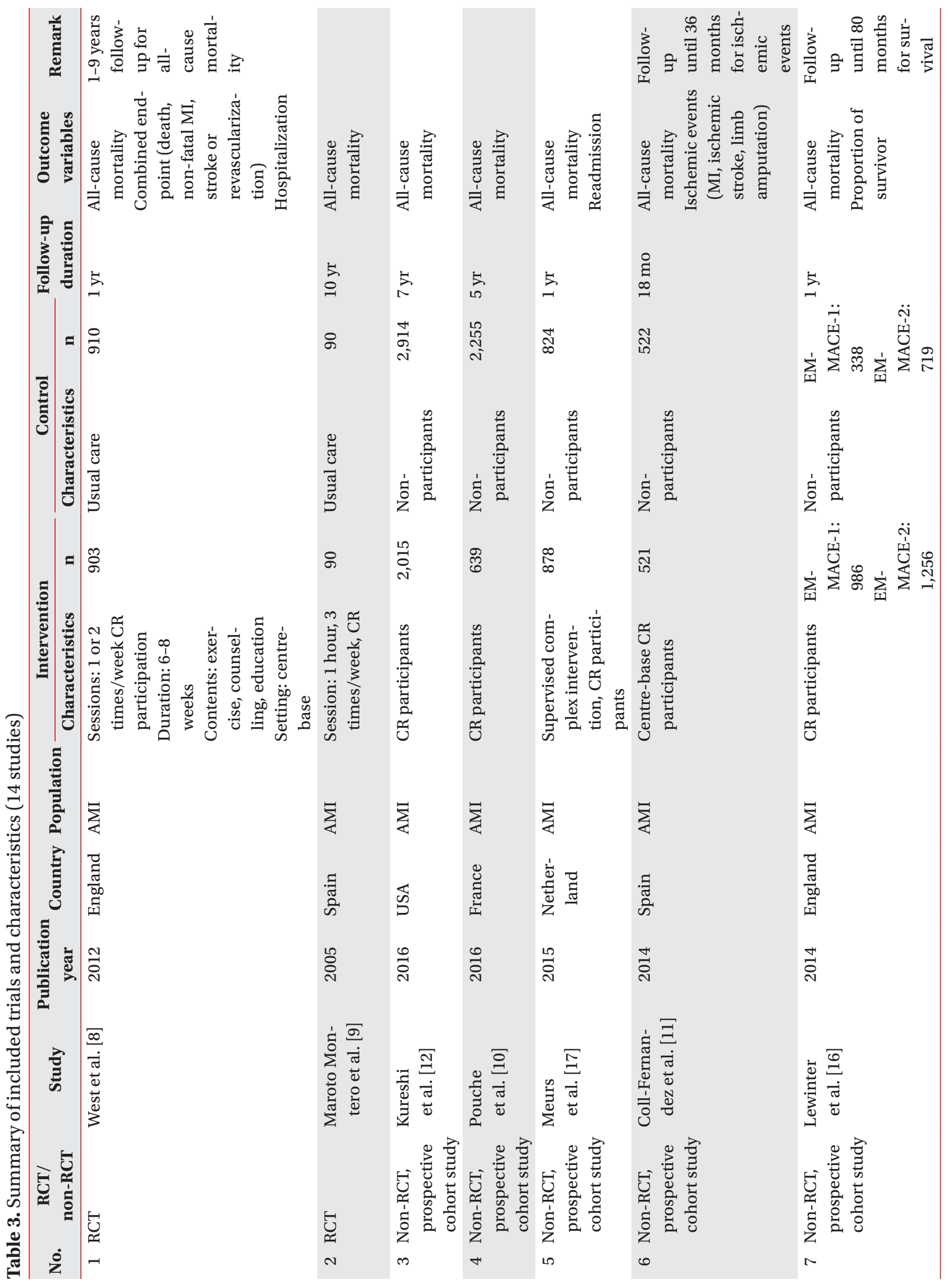




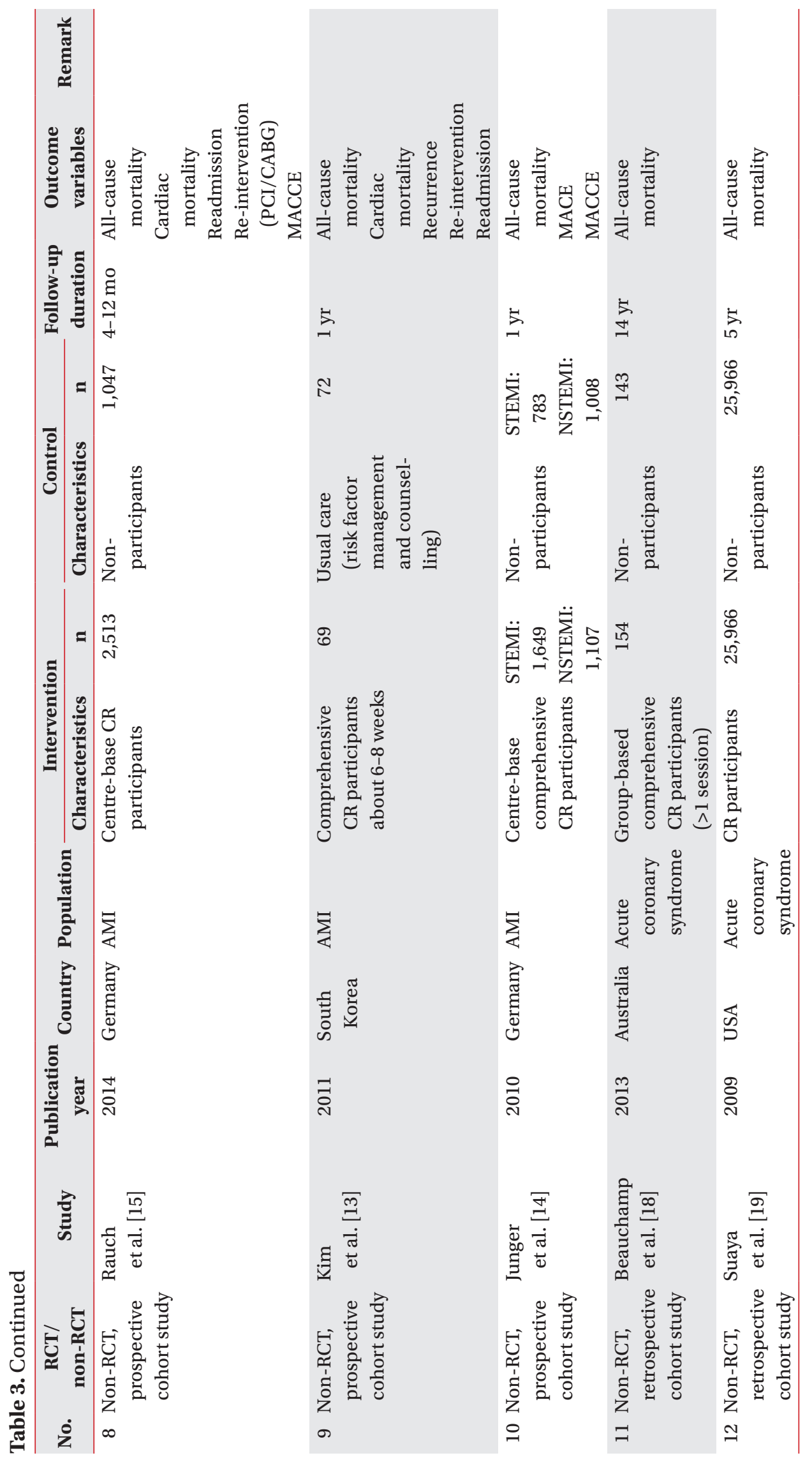




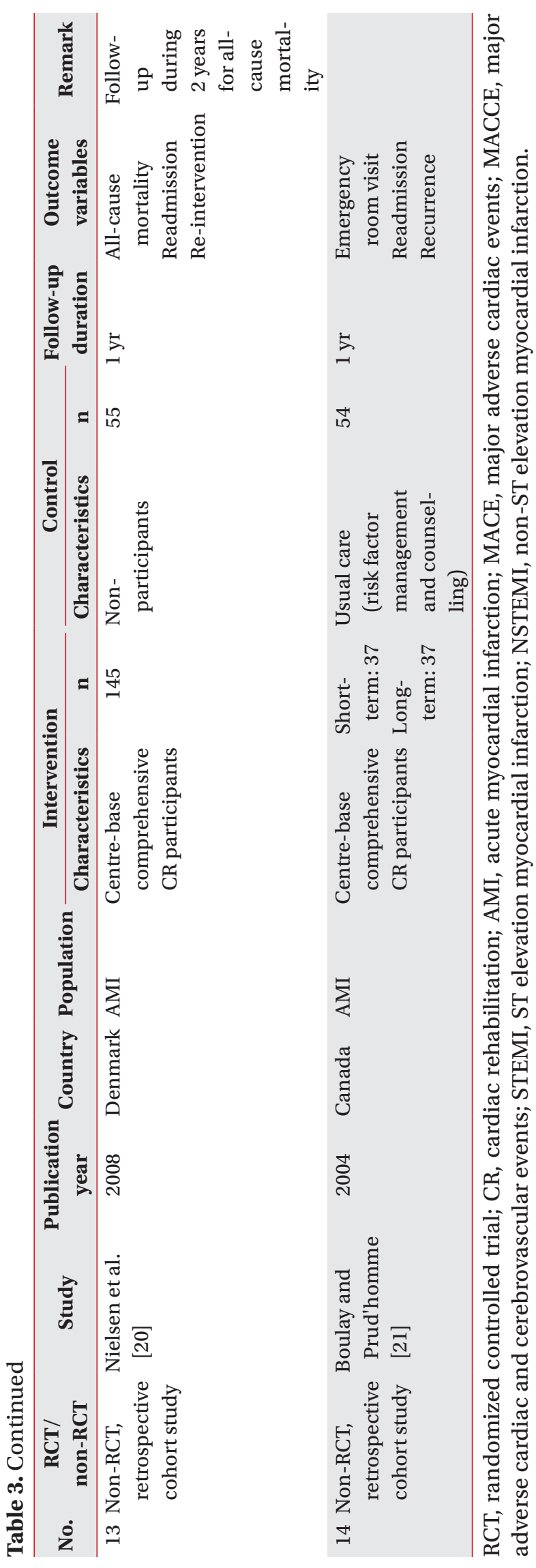

\section{Risk of bias based on study designs $R C T$}

One RCT did not report adequate details to enable a complete assessment of the potential risk of bias [8]. Among evaluation categories, performance bias was not clearly reported in any of the studies. In particular, there was a high risk of detection bias in one study, while it was not clearly reported in another study (Fig. 2).

\section{Non-RCTs}

We evaluated the risk of bias for a cohort study using the RoBANS tool, which was developed in the Republic of Korea. We divided the cohort studies into prospective and retrospective cohort studies. Next, we evaluated the comparability of participants, selection of participants, confounding variables, measurement of exposure, blinding of outcome assessment, outcome evaluation, incomplete outcome data, and selective reporting for each cohort study. Several prospective studies showed a high risk or unclear risk in comparability, selection of participants, confounding variables, measurement of exposure, and outcome evaluation. Some retrospective studies showed an unclear risk of bias for incomplete outcome data (Fig. 3). Table 1 describes the outcome variables according to the study designs. Meta-analysis was conducted using the outcome variables.

\section{Meta-analysis of RCTs \\ All-cause mortality}

Two studies used all-cause mortality as a measure of the effectiveness of CR for 9-10 years after AMI $[8,9]$. Pooled results across studies showed that CR participation was associated with a decrease in all-cause mortality after AMI, compared with no CR participation ( $R R=0.72$; 95\% CI, 0.34-1.57) (Fig. 4). Heterogeneity was calculated with $\mathrm{I}^{2}$, which was $69 \%$ (Fig. 4, Table 5).

\section{Readmission}

There was only 1 study that reported the effect of CR on the rate of readmission [8]. West et al. [8] showed no significant difference between CR participants and non-CR participants for 1 year after enrollment (Table 5).

\section{Meta-analysis of non-RCTs All-cause mortality}

The meta-analysis showed a high heterogeneity score 
Table 4. Outcome variables according to study designs

\begin{tabular}{|c|c|c|c|}
\hline \multirow{2}{*}{ Outcomes variable } & \multicolumn{3}{|c|}{ Study design } \\
\hline & Randomized controlled trial & Prospective cohort & Retrospective cohort \\
\hline Cardiac mortality & & $\mathrm{O}$ & \\
\hline All-cause mortality & $\mathrm{O}$ & $\mathrm{O}$ & $\mathrm{O}$ \\
\hline Re-intervention & & $\mathrm{O}$ & $\mathrm{O}$ \\
\hline Recurrence & & & $\mathrm{O}$ \\
\hline Readmission & $\mathrm{O}$ & $\mathrm{O}$ & $\mathrm{O}$ \\
\hline Ischemic event & & & $\mathrm{O}$ \\
\hline Major adverse cardiac events & & $\mathrm{O}$ & \\
\hline $\begin{array}{l}\text { Major adverse cardiac and } \\
\text { cerebrovascular events }\end{array}$ & & $\mathrm{O}$ & \\
\hline Emergency room visit & & & $\mathrm{O}$ \\
\hline
\end{tabular}

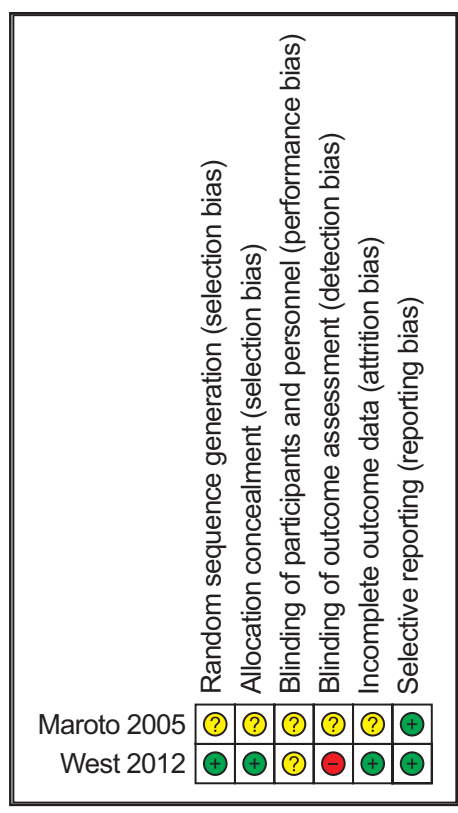

Fig. 2. Summary of risk of bias of the randomized controlled study.

among studies $\left(\mathrm{I}^{2}=93 \%\right)$. We attempted to explore the reason for high heterogeneity using meta-regression. Meta-regression analysis also revealed no significant covariates including age, sex, country, and follow up duration. Hence, we conducted a qualitative analysis of the all-cause mortality among non-RCTs.

The all-cause mortality was reported in 8 prospective cohort studies [10-17] and in 3 retrospective cohort studies [18-20]. Kureshi et al. [12] reported a significant decrease in all-cause mortality in the group that participated in CR compared to that which did not, for 7 years (hazard ratio $[\mathrm{HR}]=0.59$; 95\% CI, 0.46-0.75). Table 5 de- scribes the outcomes for mortality according to the study designs. Rauch et al. [15] reported the outcomes about cardiac mortality. In CR groups, there was a significant low odds ratio (OR) for cardiac mortality, 4 to 12 months after AMI (OR=0.43; 95\% CI, 0.23-0.79). Table 5 shows the detailed data.

\section{Re-intervention}

Data of re-intervention was reported by 2 prospective cohort studies $[13,15]$ and 1 retrospective cohort study [20]. The CR group had a higher rate of re-intervention compared to that in the non-CR group ( $\mathrm{RR}=1.25$; 95\% CI, 0.75-2.07) (Fig. 5).

\section{Recurrence}

The data of recurrence after AMI was reported by 3 prospective cohort studies $[11,13,15]$ and 1 retrospective cohort study [21]. The meta-analysis showed a significantly lower rate of recurrence in the CR group compared to that in the non-CR group (RR=0.42; 95\% CI, 0.25-0.71) (Fig. 6).

\section{Readmission}

The data about readmission was reported in 3 prospective cohort studies $[13,15,17]$ and in 2 retrospective studies $[20,21]$. The CR group showed a lower rate of readmission compared to that in the non-CR group; however, the difference was not significant $(\mathrm{RR}=0.92 ; 95 \% \mathrm{CI}$, 0.81-1.06) (Fig. 7).

\section{MACCE}

MACCE was reported by 2 cohort studies $[14,15]$. The 
(A)
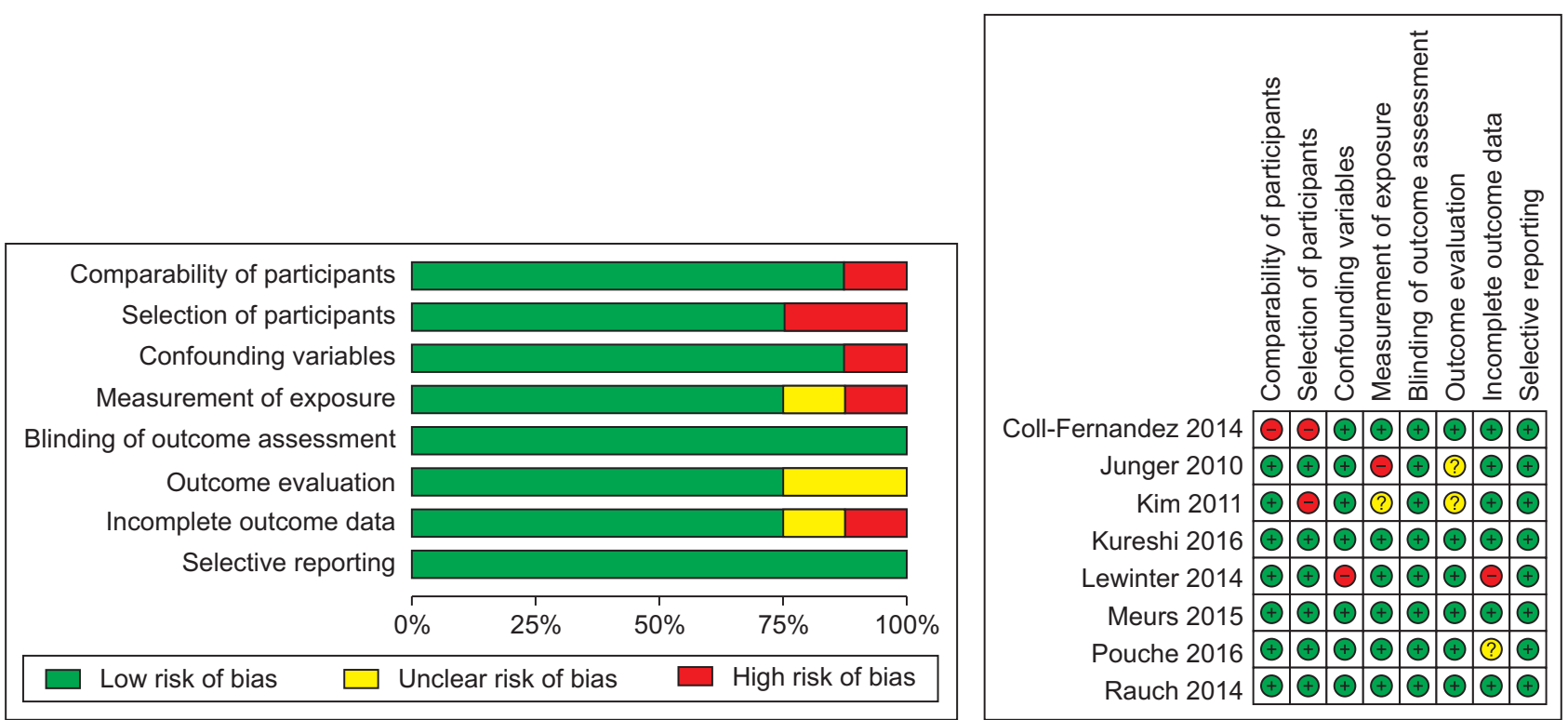

(B)
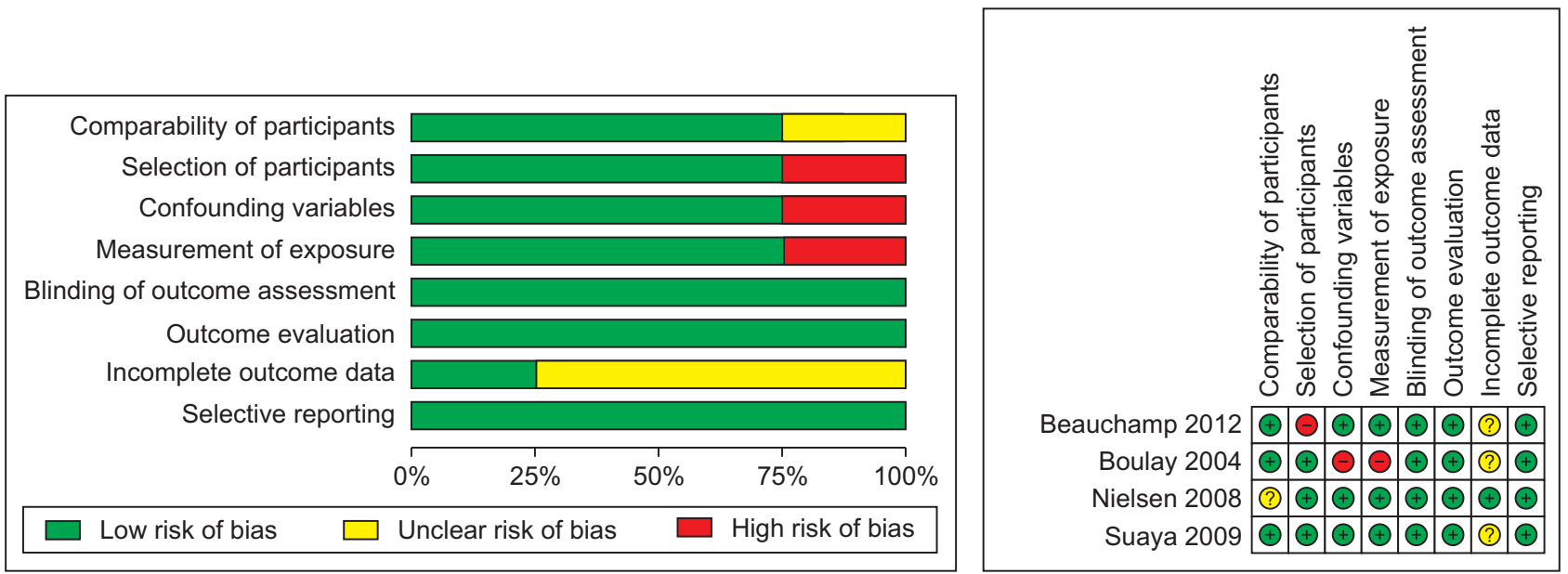

Fig. 3. Summary of risk of bias according to the study design. (A) Prospective cohort study. (B) Retrospective cohort study.

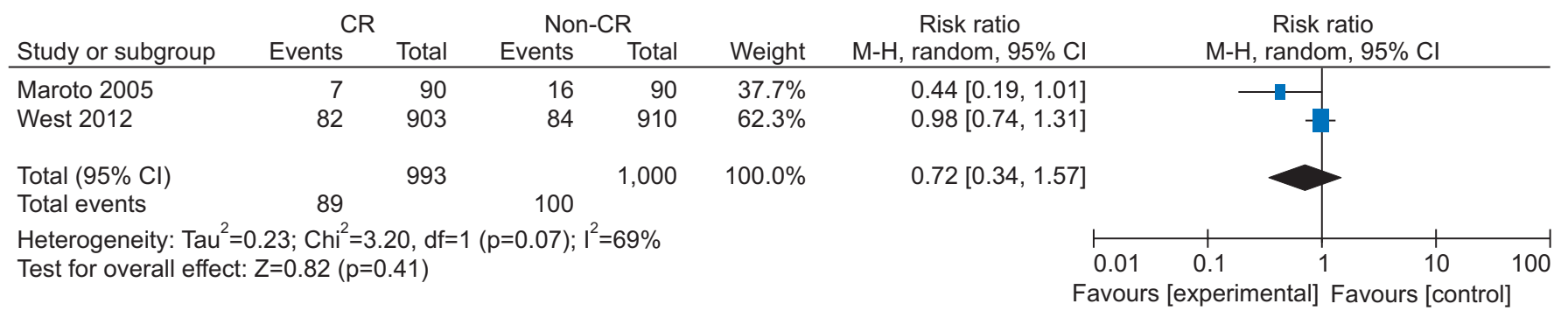

Fig. 4. Forest plot of all-cause mortality in two randomized controlled trials (RCTs). There was a decreased tendency on all-cause mortality in the cardiac rehabilitation (CR) group.

CR group showed a significantly lower rate of MACCE compared to that in the non-CR group ( $\mathrm{RR}=0.47 ; 95 \% \mathrm{CI}$, 0.40-0.55) (Fig. 8). 


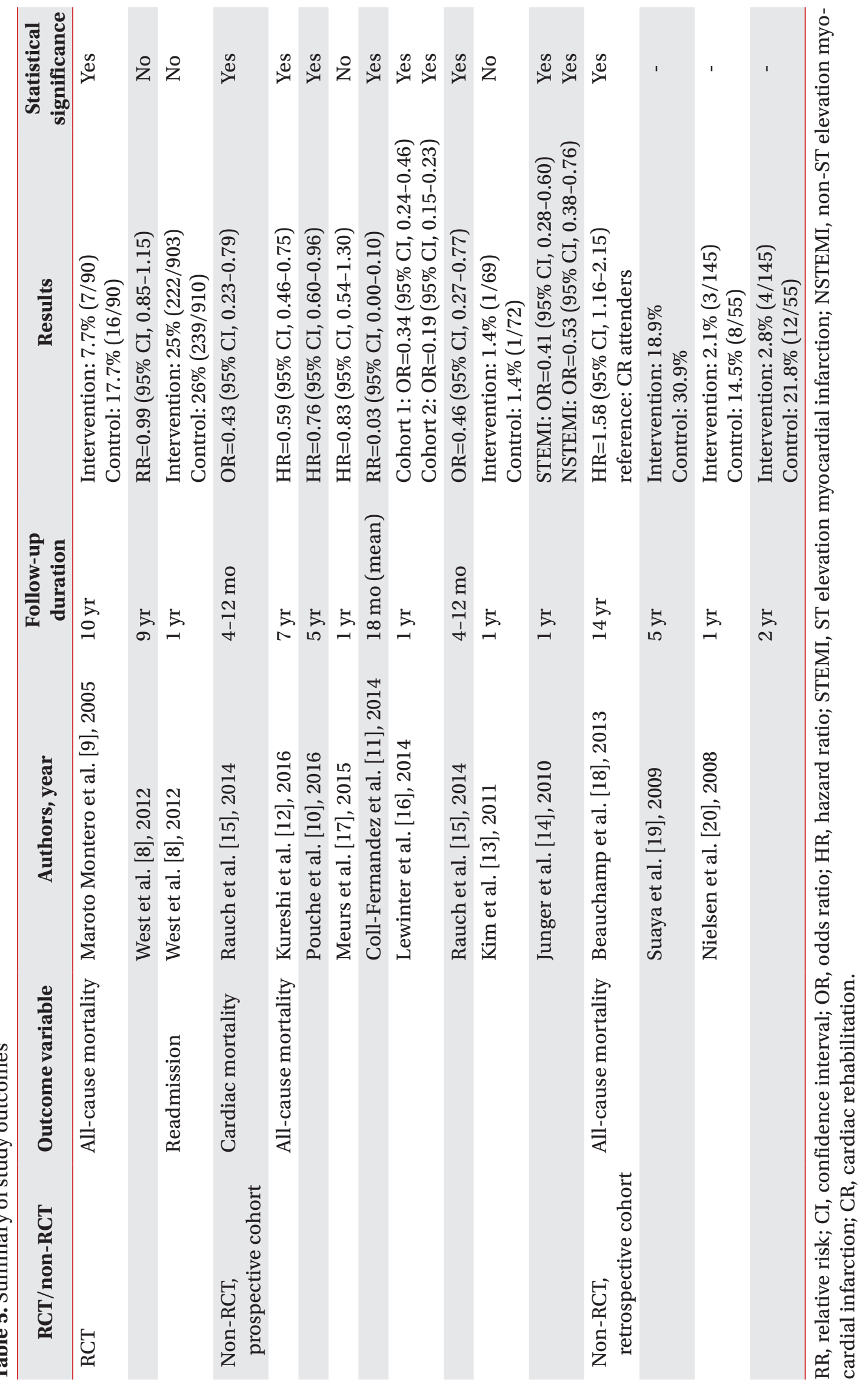




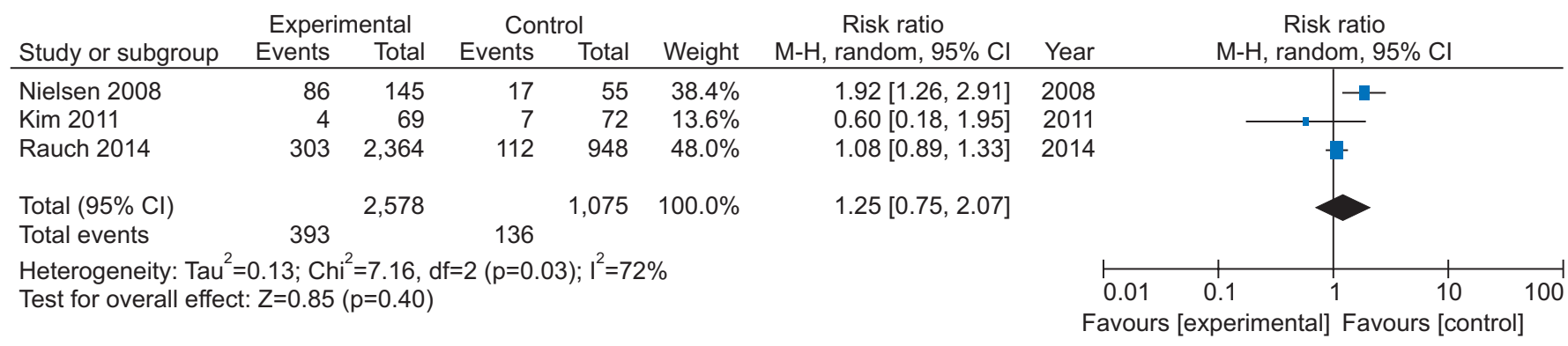

Fig. 5. Forest plot for re-intervention among non-randomized controlled trials (non-RCTs).

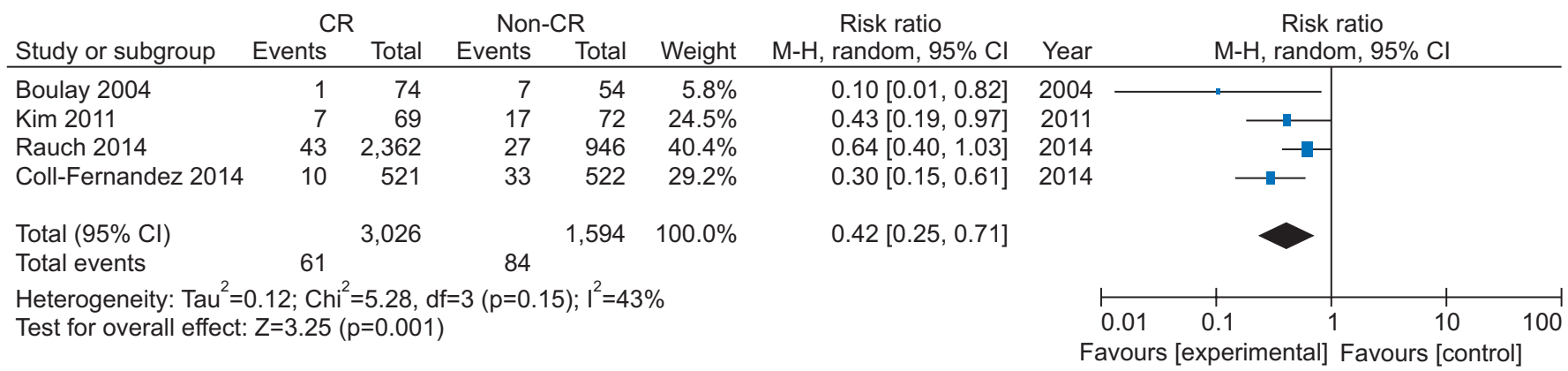

Fig. 6. Forest plot on recurrence after acute myocardial infarction among non-randomized controlled trials (non-RCTs). Cardiac rehabilitation (CR) group showed a significant decrease in recurrence.

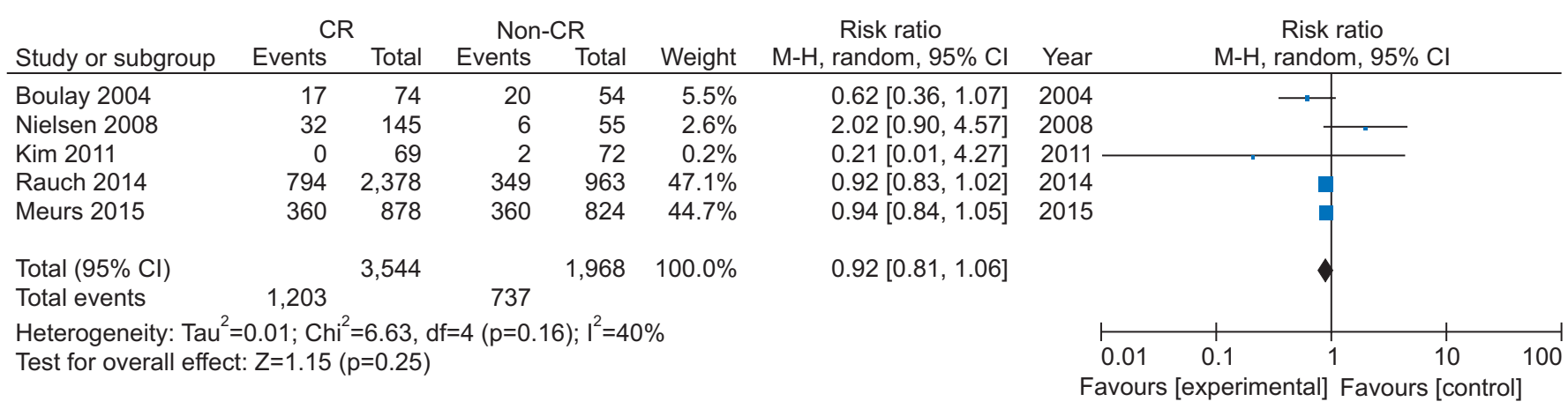

Fig. 7. Forest plot on readmission after acute myocardial infarction among non-randomized controlled trials (nonRCTs). CR, cardiac rehabilitation.

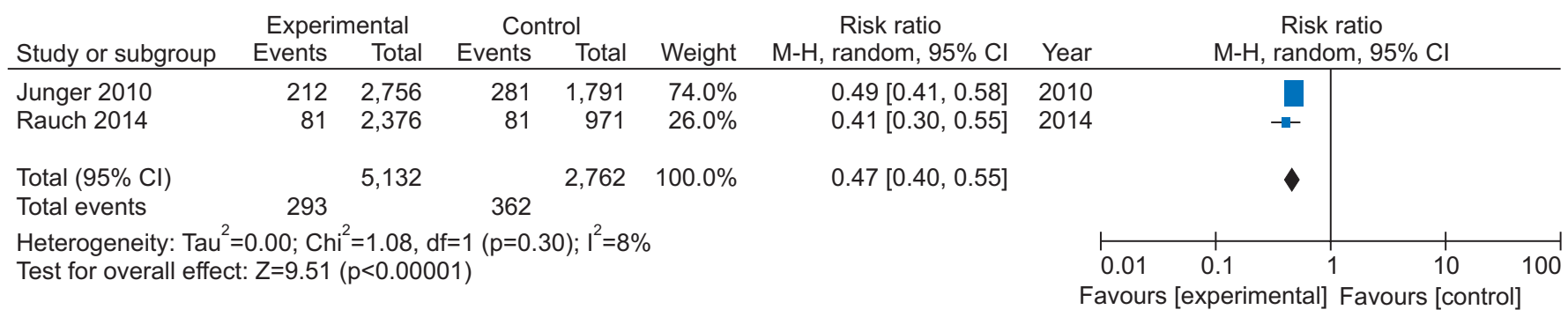

Fig. 8. Forest plot on major adverse cardiac and cerebrovascular events after acute myocardial infarction 


\section{DISCUSSION}

We conducted a systematic review and meta-analysis about the various outcomes of CR after AMI, especially during the era of primary coronary intervention. According to 14 selected studies ( 2 RCT, 8 prospective cohort studies, and 4 retrospective cohort studies) for the metaanalysis, there was a significant reduction in the rate of recurrence and MACCE in those who underwent CR compared to those who did not, reported in cohort studies and in non-RCTs. However, our analysis of the RCTs did not show a significant decrease in all-cause mortality and readmission.

In contrast to previous meta-analyses [4,5], we did not find a statistically significant reduction in all-cause mortality with CR, in the RCTs. This might be explained by the inclusion of only AMI patients, due to which our meta-analysis focused on a very specific population. According to the Cochrane studies [4,5], the population comprised those with ACS, including not only unstable angina but also AMI. We generalized the definition of CR for the inclusion criteria, as the number of studies that could be included for the meta-analysis reduced significantly with a more specific definition of CR for AMI. The general definition of CR could also affect the outcomes about impact of CR on cardiac mortality and recurrence. Hence, this generalization could show non-significance of the effect of CR. This suggests that more randomized controlled studies with well-defined CR for specific populations are necessary.

West et al. [8] conducted a large randomized controlled trial with exercise-based CR for AMI patients with 1-2 sessions per week for 6-8 weeks. However, they included relatively large populations, which might not show significantly favorable outcomes on all-cause mortality. Moreover, they did not monitor the cardiac mortality. We assumed that the negative outcome was due to the dose-dependency of CR on all-cause mortality, which was already reported by Santiago de Araujo Pio et al. [22]. According to Santiago de Araujo Pio et al. [22], West et al. [8] used low-intensity CR for AMI, in contrast to that in the study by Maroto Montero et al. [9]. This low intensity of CR could lead to non-significant results for all-cause mortality. Another factor is the population selected; we focused on CR for AMI except unstable angina. There are few RCTs that particularly explored the effect of CR in pa- tients with AMI. Hence, more studies including specific populations, intervention, and primary treatment should be conducted.

The present analysis has a few limitations. First, the poor level of reporting in the included RCTs made it difficult to evaluate the risk of bias. However, there was significant improvement in the quality of reporting in a more recent study. Nevertheless, the lack of metaanalysis about the effect of CR in patients with AMI led us to conduct this analysis. In contrast to RCTs, cohort studies showed a relatively better quality of methods. Hence, we included them for the meta-analysis. Included studies did not consistently report all outcomes relevant to this review, and events were often reported in study descriptions about dropouts or withdrawal. Our results are therefore based on small and different subsets of the overall evidence base. However, we found our results to be consistent with a previous meta-analysis about the rate of re-intervention and readmission in those who underwent CR. Finally, we sought to focus on the diagnoses of study participants as AMI on the basis of Braunwald's classification of CHD [23], to study whether the CR can affect several outcomes after AMI, including non-ST-segment elevation MI. We could not apply this specific categorization of the diagnosis due to the limited reporting by studies about the inclusion and exclusion criteria and participants' characteristics. Nevertheless, we believe this to be the most comprehensive review of evidence to date, summarizing the results of RCTs with 2,000 AMI and non-RCTs with $>20,000$ AMI patients.

In conclusion, the present study was planned and conducted to identify the effects of CR on the post-discharge prognosis of patients with AMI from two perspectives. A systematic literature review of recent articles was conducted, and a comparison between CR participation and non-participation groups among patients with AMI in Korea was performed.

The meta-analysis shows that CR reduces the risk of rehospitalization and all-cause mortality after AMI, compared to no participation in CR. This outcome was seen in non RCTs but not in RCTs. We believe that more studies are necessary for conclusive outcomes of CR on AMI with various settings and intensities. 


\section{CONFLICT OF INTEREST}

No potential conflict of interest relevant to this article was reported.

\section{ACKNOWLEDGMENTS}

This study was supported by National Evidence-based Healthcare Collaborating Agency (NECA) funded by the Ministry of Health and welfare (No. NECA-C-2017-006). We also thank Euniung Park, Jung Im Shim, and Jin-A Choi at the National Evidence-based Healthcare Collaborating Agency for their assist on searching and distributing the literatures.

\section{AUTHOR CONTRIBUTION}

Conceptualization: Kim C. Methodology: Kim C, Jee S, Kim AR, Kim W. Formal analysis: Cho S, Choi I. Funding acquisition: Kim C. Project administration: Jee S, Visualization: Choi I, Cho S. Writing - original draft: Jee S, Cho S, Writing - review and editing: Kim C, Jee S. Approval of final manuscript: all authors.

\section{SUPPLEMENTARY MATERIALS}

Supplementary materials can be found via https://doi. org/10.5535/arm.20080.

\section{REFERENCES}

1. Statistics Korea. 2015 causes of death statistics [Internet]. Daejeon, Korea: Statistics Korea; 2016 [cited 2020 Dec 31]. Available from: http://kostat.go.kr/portal/ korea/kor_nw/1/6/2/index.board?bmode $=$ read $\& b S e$ $\mathrm{q}=\& \mathrm{aSeq}=356345 \&$ pageNo=1\&rowNum $=10$ \&navCoun $\mathrm{t}=10 \&$ currPg=\&searchInfo $=\&$ sTarget $=$ title \& $\mathrm{sTx}=$.

2. Kweon S, Sohn MK, Jeong JO, Kim S, Jeon H, Lee H, et al. Quality of life and awareness of cardiac rehabilitation program in people with cardiovascular diseases. Ann Rehabil Med 2017;41:248-56.

3. WHO Expert Committee on Rehabilitation of Patients with Cardiovascular Diseases \& World Health Organization. Rehabilitation of patients with cardiovascular diseases: report of a WHO Expert Committee (meeting held in Geneva from 23 to 29 July 1963). Geneva, Swit- zerland: World Health Organization; 1964.

4. Anderson L, Oldridge N, Thompson DR, Zwisler AD, Rees K, Martin N, et al. Exercise-based cardiac rehabilitation for coronary heart disease: Cochrane systematic review and meta-analysis. J Am Coll Cardiol 2016;67:1-12.

5. Rauch B, Davos CH, Doherty P, Saure D, Metzendorf MI, Salzwedel A, et al. The prognostic effect of cardiac rehabilitation in the era of acute revascularisation and statin therapy: a systematic review and meta-analysis of randomized and non-randomized studies. The Cardiac Rehabilitation Outcome Study (CROS). Eur J Prev Cardiol 2016;23:1914-39.

6. Kim SY, Park JE, Lee YJ, Seo HJ, Sheen SS, Hahn S, et al. Testing a tool for assessing the risk of bias for nonrandomized studies showed moderate reliability and promising validity. J Clin Epidemiol 2013;66:408-14.

7. Balshem H, Helfand M, Schunemann HJ, Oxman AD, Kunz R, Brozek J, et al. GRADE guidelines: 3. Rating the quality of evidence. J Clin Epidemiol 2011;64:4016.

8. West RR, Jones DA, Henderson AH. Rehabilitation after myocardial infarction trial (RAMIT): multi-centre randomised controlled trial of comprehensive cardiac rehabilitation in patients following acute myocardial infarction. Heart 2012;98:637-44.

9. Maroto Montero JM, Artigao Ramirez R, Morales Duran MD, de Pablo Zarzosa C, Abraira V. Cardiac rehabilitation in patients with myocardial infarction: a 10year follow-up study. Rev Esp Cardiol 2005;58:1181-7.

10. Pouche M, Ruidavets JB, Ferrieres J, Iliou MC, Douard H, Lorgis L, et al. Cardiac rehabilitation and 5 -year mortality after acute coronary syndromes: the 2005 French FAST-MI study. Arch Cardiovasc Dis 2016;109:178-87.

11. Coll-Fernandez R, Coll R, Pascual T, Sanchez MunozTorrero JF, Sahuquillo JC, Manzano L, et al. Cardiac rehabilitation and outcome in stable outpatients with recent myocardial infarction. Arch Phys Med Rehabil 2014;95:322-9.

12. Kureshi F, Kennedy KF, Jones PG, Thomas RJ, Arnold SV, Sharma P, et al. Association between cardiac rehabilitation participation and health status outcomes after acute myocardial infarction. JAMA Cardiol 2016;1:980-8.

13. Kim C, Kim DY, Moon CJ. Prognostic influences of 
cardiac rehabilitation in Korean acute myocardial infarction patients. Ann Rehabil Med 2011;35:375-80.

14. Junger C, Rauch B, Schneider S, Liebhart N, Rauch $\mathrm{G}$, Senges J, et al. Effect of early short-term cardiac rehabilitation after acute ST-elevation and non-STelevation myocardial infarction on 1-year mortality. Curr Med Res Opin 2010;26:803-11.

15. Rauch B, Riemer T, Schwaab B, Schneider S, Diller F, Gohlke H, et al. Short-term comprehensive cardiac rehabilitation after AMI is associated with reduced 1-year mortality: results from the OMEGA study. Eur J Prev Cardiol 2014;21:1060-9.

16. Lewinter C, Bland JM, Crouch S, Doherty P, Lewin RJ, Kober $\mathrm{L}$, et al. The effect of referral for cardiac rehabilitation on survival following acute myocardial infarction: a comparison survival in two cohorts collected in 1995 and 2003. Eur J Prev Cardiol 2014;21:163-71.

17. Meurs M, Burger H, van Riezen J, Slaets JP, Rosmalen JG, van Melle JP, et al. The association between cardiac rehabilitation and mortality risk for myocardial infarction patients with and without depressive symptoms. J Affect Disord 2015;188:278-83.
18. Beauchamp A, Worcester M, Ng A, Murphy B, Tatoulis J, Grigg L, et al. Attendance at cardiac rehabilitation is associated with lower all-cause mortality after 14 years of follow-up. Heart 2013;99:620-5.

19. Suaya JA, Stason WB, Ades PA, Normand SL, Shepard DS. Cardiac rehabilitation and survival in older coronary patients. J Am Coll Cardiol 2009;54:25-33.

20. Nielsen KM, Faergeman O, Foldspang A, Larsen ML. Cardiac rehabilitation: health characteristics and socio-economic status among those who do not attend. Eur J Public Health 2008;18:479-83.

21. Boulay P, Prud'homme D. Health-care consumption and recurrent myocardial infarction after 1 year of conventional treatment versus short- and long-term cardiac rehabilitation. Prev Med 2004;38:586-93.

22. Santiago de Araujo Pio C, Marzolini S, Pakosh M, Grace SL. Effect of cardiac rehabilitation dose on mortality and morbidity: a systematic review and metaregression analysis. Mayo Clin Proc 2017;92:1644-59.

23. Mann DL, Zipes DP, Libby P, Bonow RO. Braunwald's heart disease : a textbook of cardiovascular medicine. 10th ed. Philadelphia, PA: Elsevier/Saunders; 2015. 